\title{
Gallium Ga 68-DOTATATE
}

National Cancer Institute

\section{Source}

National Cancer Institute. Gallium Ga 68-DOTATATE. NCI Thesaurus. Code C97661.

A radioconjug ate consisting of the somatostatin analogue tyrosine-3-octreotate (Tyr3octreotate or TATE) labeled with the positron emission tomography (PET) tracer gallium Ga 68 via the macrocyclic chelating agent dodecanetetraacetic acid (DOTA), which may be used as a somatostatin receptor (SSTR) imaging agent in conjunction with PET to image neuroendocrine tumors (NETs). Gallium Ga 68-DOTATATE binds to SSTRs, with a much higher affinity for type 2 SSTR, present on the cell membranes of many types of NETs. This allows for visualization of SSTR-positive cells upon imaging. SST R subtypes have been shown to be present in large numbers on NET s and their metastases, while most other normal tissues express low levels of SSTR subtypes. 